\title{
Incidence, clinical features and diagnosis of cicatrising conjunctivitis in Australia and New Zealand
}

\author{
Samantha Bobba ${ }^{1} \cdot$ Connor Devlin ${ }^{2} \cdot$ Nick Di Girolamo $^{3} \cdot$ Denis Wakefield ${ }^{1,3} \cdot$ Peter McCluskey $^{2,4} \cdot$ Elsie Chan $^{5}$. \\ Mark Daniell ${ }^{5}$. Stephanie Watson ${ }^{1,2,4}$
}

Received: 15 February 2018 / Revised: 1 May 2018 / Accepted: 21 May 2018 / Published online: 19 June 2018

(c) The Royal College of Ophthalmologists 2018

\begin{abstract}
Aims This study aimed to determine the incidence, clinical features and management of cicatrising conjunctivitis in Australia and New Zealand, also enabling comparison with data from the United Kingdom.

Methods A prospective surveillance study was conducted over 17 months via the Australian and New Zealand Ophthalmic Surveillance Unit with a one-year follow-up period. Practicing ophthalmologists on the Surveillance Unit's database were asked to report recently diagnosed cases of cicatrising conjunctivitis on a monthly basis. Initial and follow-up questionnaires were sent to ophthalmologists who had reported positive cases to obtain demographic and clinical data. The minimum incidence of cicatrising conjunctivitis was calculated based on cases reported during the study period and from population data.

Results During the 17-month study period (December 2011-April 2013), 56 cases of cicatrising conjunctivitis were reported. Data was obtained for 35 cases $(62 \%)$ with a mean age of 74 years (range, 28-94 years). The most common aetiologies were ocular mucus membrane pemphigoid ( $n=18$ cases, $51.4 \%)$, Stevens-Johnson Syndrome $(n=3,8.6 \%)$ and graft versus host disease $(n=3,8.6 \%)$. The minimum incidence of cicatrising conjunctivitis in Australia and New Zealand was 1.5 per million, comparable to incidence data from the United Kingdom.

Conclusions This study is the first to prospectively record the incidence of cicatrising conjunctivitis in Australia and New Zealand and the second worldwide. It provides novel data on demographics and management of cicatrising conjunctivitis, as reported by treating ophthalmologists.
\end{abstract}

Electronic supplementary material The online version of this article (https://doi.org/10.1038/s41433-018-0155-7) contains supplementary material, which is available to authorized users.

Samantha Bobba

Samantha.bobba@gmail.com

$\bowtie$ Stephanie Watson

Stephanie.watson@sydney.edu.au

1 Prince of Wales Clinical School, University of New South Wales, High Street, Randwick, Sydney, NSW 2031, Australia

2 Sydney Eye Hospital, Sydney, Australia, 8 Macquarie Street, Sydney, NSW 2000, Australia

3 School of Medical Sciences, University of New South Wales, Kensington, Sydney, NSW 2052, Australia

4 Save Sight Institute, University of Sydney, 8 Macquarie Street, Sydney, NSW 2000, Australia

5 Royal Victorian Eye and Ear Hospital, Centre for Eye Research Australia, University of Melbourne, 32 Gisbourne Street, East Melbourne, VIC 3002, Australia

\section{Introduction}

Cicatrising conjunctivitis is a rare, potentially sightthreatening condition characterised by persistent conjunctival inflammation and progressive conjunctival scarring [1]. Patients may experience severe ocular discomfort secondary to corneal ulceration and ocular surface damage. Blinding keratopathy may result from infectious keratitis or chronic limbitis and subsequent limbal stem cell failure [2]. This condition can also be associated with ocular or systemic diseases, such as atopic keratoconjunctivitis or mucous membrane pemphigoid (MMP), respectively.

Ocular mucous membrane pemphigoid (OcMMP), previously known as ocular cicatricial pemphigoid, is the most common underlying aetiology of cicatrising conjunctivitis, comprising approximately $60 \%$ of cases $[1,3]$. It accompanies $70 \%$ of cases of MMP, an immune-mediated disease involving the mucus membranes and the skin to a lesser extent [4], and is characterised by recurrent blisters and 
bullae leading to scar formation [1, 2, 5]. Clinical signs of OcMMP include loss of plica, subepithelial fibrosis, progressive shrinkage of the fornices, trichiasis, cicatrical entropion, symblepharon formation and anklyblepharon in severe cases [1, 3, 5]. Prognosis can be significantly improved with systemic immunosuppression [6-8].

Other causes of cicatrising conjunctivitis are varied and extensive, and include Stevens-Johnson Syndrome/Toxic Epidermal Necrolysis (SJS/TEN), linear IgA disease, epidermolysis bullosa acquistia, drug-induced cicatrising conjunctivitis, graft versus host disease (GVHD), mucocutaneous paraneoplastic disorders, atopic keratoconjunctivitis, ocular rosacea, and ocular surface squamous neoplasia [1, 9-11]. Late diagnosis of cicatrising conjunctivitis may require more aggressive immunotherapy for disease control, with potentially more treatment-related adverse effects. The rarity of the condition and difficulties in distinguishing between its underlying causes may contribute to delayed diagnosis and treatment [1].

This surveillance study was based upon a prospective nation-wide survey of cicatrising conjunctivitis conducted in the United Kingdom [12]. It was conducted in association with the Australian and New Zealand Ophthalmic Surveillance Unit (ANZOSU), utilising a similar methodology to the recently published incidence study of limbal stem cell deficiency (LSCD) [13]. It is the first national study to document the incidence of cicatrising conjunctivitis and its underlying aetiologies in Australia and New Zealand and the second such study worldwide.

In the United Kingdom, Radford and colleagues (2012) calculated a minimum incidence of $1.3 /$ million for cicatrising conjunctivitis, including 0.8/million for OcMMP [12]. Documenting the incidence and nature of cicatrising conjunctivitis in Australia and New Zealand allows for comparison of incidence, diagnostic approaches and management with the United Kingdom and may also facilitate the establishment of local specialist clinics based on patient distribution.

\section{Methods}

\section{Surveillance study design}

A prospective study was undertaken in collaboration with the ANZOSU over a 17-month period between December 2011 and April 2013, with a 12-month follow-up period. The ANZOSU was associated with the Royal Australian and New Zealand College of Ophthalmologists (RANZCO), and based upon the structure of the British Ophthalmic Surveillance Unit [12, 14]. The study was approved by the South Eastern Sydney Local Health District Low/Negligible Risk Committee (SESLHD
HREC-12/306) and conducted in accordance with the Tenets of the Declaration of Helsinki.

Cicatrising conjunctivitis was defined as conjunctival inflammation associated with scarring [1]. Stevens-Johnson Syndrome/Toxic Epidermal Necrolysis (SJS/TEN) was defined as conjunctival inflammation after an acute vesicobullous disorder with skin and mucus membrane involvement $[12,15]$. Ocular MMP was defined as having the characteristic phenotype of a conjunctival fibrosis pattern with or without other mucous membrane or skin involvement after other causes had been excluded and/or immunofluorescence or histopathology results suggestive of OcMMP on conjunctival biopsy testing [1, 4, 12].

Ophthalmologists on the ANZOSU's database were contacted by the Surveillance Unit on a monthly basis and asked to report newly diagnosed cases of cicatrising conjunctivitis. They were assigned a study protocol number for each reported case and asked to complete an initial questionnaire, with follow-up data sought 12 months after initial diagnosis. Patients with a history of trachoma, trauma or an acute infectious membranous conjunctivitis were excluded.

\section{Data collection}

To allow for comparison of data, the inclusion/exclusion criteria and design of the initial and follow-up questionnaires (Appendix I, Appendix II) were developed in collaboration with senior ophthalmologists and leading ocular scientists at Moorfields Eye Hospital, based upon their incidence study of cicatrising conjunctivitis in the United Kingdom [12]. The questionnaires have been validated on a retrospective cohort [2].

Reporting ophthalmologists were contacted by the principal investigators with an initial questionnaire to collect demographic and clinical data, including gender, postcode, age, medical history, aetiology of cicatrising conjunctivitis, disease onset, method of diagnosis, extraocular manifestations, visual acuity, degree of cicatrisation, extent of conjunctival inflammation, keratopathy and the management initiated. Questionnaires were also sought at 12-month follow-up and included similar clinical details in addition to data on changes to the underlying diagnosis, further investigations and alterations to treatment. Data sheets were collated, information de-identified and results entered into an electronic database for analysis.

\section{Statistical analysis}

Using population data, minimum incidence rates were calculated for cicatrising conjunctivitis and its most common aetiologies by extrapolating from the number of cases reported during the 17-month study period. This was compared to incidence data from internationally published 
Table 1 Incidence of CC and/or SJS in Australia and New Zealand

\begin{tabular}{|c|c|c|c|}
\hline $\begin{array}{l}\text { Geographical regions in } \\
\text { ANZ (Dec 2011-April } \\
\text { 2013) }\end{array}$ & $\begin{array}{l}\text { Number of } \\
\text { reported } \\
\text { cases (\% to } \\
\text { nearest } \\
\text { decimal) }\end{array}$ & Population $^{\mathrm{a}}$ & $\begin{array}{l}\text { Incidence per } \\
\text { million (adjusted } \\
\text { per annum) }\end{array}$ \\
\hline \multicolumn{4}{|c|}{ State capital cities with reported cases of CC and recorded geographical dat } \\
\hline Sydney, NSW & $3(8.8 \%)$ & $4,391,674$ & 0.5 \\
\hline Melbourne, VIC & $10(29.4 \%)$ & $3,999,982$ & 1.8 \\
\hline Perth, WA & $1(2.9 \%)$ & $1,830,000$ & 0.4 \\
\hline Adelaide, SA & $2(5.9 \%)$ & $1,263,000$ & 1.1 \\
\hline Canberra, ACT & $1(2.9 \%)$ & 367,800 & 1.9 \\
\hline Non-metropolitan NSW & $7(20.6 \%)$ & $2,818,326$ & 1.8 \\
\hline South Coast & $1(2.9 \%)$ & 71,551 & 9.8 \\
\hline $\begin{array}{l}\text { Central Coast (Gosford } \\
\text { and Wyong) }\end{array}$ & $2(5.9 \%)$ & 333,119 & 4.2 \\
\hline Newcastle & $2(5.9 \%)$ & 308,308 & 4.6 \\
\hline Lismore/Byron Bay & $2(5.9 \%)$ & 49,468 & 28.5 \\
\hline $\begin{array}{l}\text { Non-metropolitan } \\
\text { Victoria }\end{array}$ & $4(11.8 \%)$ & $1,300,018$ & 2.2 \\
\hline Mornington Peninsula & $1(2.9 \%)$ & 144,608 & 4.8 \\
\hline Geelong & $1(2.9 \%)$ & 215,837 & 3.2 \\
\hline Ballarat & $1(2.9 \%)$ & 95,185 & 7.4 \\
\hline Horsham & $1(2.9 \%)$ & 15,894 & 44.4 \\
\hline Non-metropolitan WA & $2(5.9 \%)$ & 520,000 & 2.7 \\
\hline Rockingham & $1(2.9 \%)$ & 109,415 & 6.5 \\
\hline Augusta & $1(2.9 \%)$ & 1,292 & 546 \\
\hline Queensland & $2(5.9 \%)$ & $4,332,739$ & 0.3 \\
\hline Sunshine Coast & $1(2.9 \%)$ & 267,241 & 2.6 \\
\hline Redland City & $1(2.9 \%)$ & 143,628 & 4.9 \\
\hline Northern territory & $1(2.9 \%)$ & 211,945 & 3.3 \\
\hline Alice Springs & $1(2.9 \%)$ & 25,186 & 28 \\
\hline New Zealand & $1(2.9 \%)$ & $4,242,048^{a}$ & 0.2 \\
\hline Dunedin & $1(2.9 \%)$ & 120,246 & 5.8 \\
\hline \multirow[t]{3}{*}{$\begin{array}{l}\text { Australia and New } \\
\text { Zealand }\end{array}$} & $\begin{array}{l}\text { State Capital } \\
\text { Cities: } 17\end{array}$ & $13,613,212$ & 0.9 \\
\hline & $\begin{array}{l}\text { Non- } \\
\text { Metropoli- } \\
\text { tan }^{b}: 17\end{array}$ & $12,136,553$ & 1.0 \\
\hline & $\begin{array}{ll}\text { Total: } & 34 \\
& 56^{c}\end{array}$ & $25,749,765$ & $\begin{array}{l}0.9 \\
1.5^{\mathrm{c}}\end{array}$ \\
\hline
\end{tabular}

Only reported cases are recorded in the table. No cases were reported in the state capital cities of Darwin, Brisbane, Hobart or Auckland. No cases were reported in Tasmania or non-metropolitan South Australia ABS Australian Bureau of Statistics, ACT Australian Capital Territory, ANZ Australia and New Zealand, NSW New South Wales, NT Northern Territory, SA South Australia, WA Western Australia

aPopulation data is based on the June 2011 ABS Census data for Australia and 2013 Census data for NZ due to cancellation of the 2011 Census by the NZ government

${ }^{b}$ Non-Metropolitan areas are defined as outside a state capital city

Inclusive of 34 cases with recorded postcodes and an additional 22 cases reported to the ANZOSU with unrecorded geographical data

reports [12, 16, 17]. Demographic (e.g. age, geographical location and gender) and clinical (e.g. aetiology of cicatrising conjunctivitis, diagnostic approach, diagnostic delay and short-term progression) data were analysed.

\section{Results}

\section{Response rates, aetiology and incidence data}

Fifty-six cases of cicatrising conjunctivitis were reported to the ANZOSU by 47 different ophthalmologists; however data were unavailable for 20 patients and erroneous for one patient $(n=21 / 56,36 \%)$. Of the remaining 35 cases, 18 $(51.4 \%)$ were diagnosed with proven or presumed OcMMP. Fifty-five percent of these patients $(n=10 / 18)$ had extraocular manifestations. Other aetiologies included SJS $(n=3$, $8.6 \%)$, GVHD $(n=3,8.6 \%)$, medication-induced cicatrising conjunctivitis $(n=2,5.7 \%)$, TEN $(n=1,2.9 \%)$, linear IgA disease $(n=1,2.9 \%)$ and ocular lichen planus $(n=1$, 2.9\%). One patient had both GVHD and SJS. Twelvemonth follow-up data was received for 27 of the 35 patients (77\%), which comprised 16 of the 18 cases initially diagnosed as OcMMP (89\%) and 3 of the 4 (75\%) SJS/TEN cases. Based on population data and the 56 cases reported in total over the 17-month study period, the incidence of cicatrising conjunctivitis in Australia and New Zealand was extrapolated to $1.5 /$ million/annum. Excluding cases with unrecorded underlying aetiologies from analysis, the incidence of OcMMP was 0.7/million/annum, 0.1/million/ annum for SJS/TEN and 0.4/million/annum for other causes of cicatrising conjunctivitis.

\section{Patient demographics and geographical variations}

Based on the 35 cases with correctly completed questionnaires, the mean age of patients was $74 \pm 16$ years, (range, 28-94 years), with a slight predominance of males reported (females, $n=15,43 \%$; males, $n=20,57 \%$ ). Patients with a diagnosis of SJS/TEN had a younger mean age of $43 \pm 10$ years (range, 28-53 years). The incidence of cicatrising conjunctivitis was not significantly different between state capital cities (0.9/ million) and nonmetropolitan areas (1.0/million). There was, however, significant geographical variation in the incidence of cicatrising conjunctivitis across New Zealand and Australia (Table 1, Table 2). In comparing Australia's two most populated state capital cities, Melbourne had a significantly higher incidence of cicatrising conjunctivitis at $1.8 /$ million compared to Sydney at $0.5 /$ million (see Table 1). New Zealand and Queensland had relatively low incidence levels (0.2/million and $0.3 /$ million respectively), whilst the Northern Territory had the highest incidence of any state or territory at 3.3/million. Of the reporting ophthalmologists, a larger proportion of their clinics were located in state capital cities $(n=37 / 56,66 \%)$ compared with non-metropolitan regions $(n=19 / 56,34 \%)$. 
Table 2 The geographical distribution of ophthalmology clinics/ centres reporting cases of CC and/or SJS in ANZ

\begin{tabular}{|c|c|}
\hline Location & $\begin{array}{l}\text { Number of } \\
\text { reporting } \\
\text { centres }(\%)\end{array}$ \\
\hline \multicolumn{2}{|l|}{ New South Wales } \\
\hline Sydney & $11(26 \%)$ \\
\hline Non-metropolitan & $3(7 \%)$ \\
\hline \multicolumn{2}{|l|}{ Victoria } \\
\hline Melbourne & $8(19 \%)$ \\
\hline Non-metropolitan & $2(5 \%)$ \\
\hline \multicolumn{2}{|l|}{ Queensland } \\
\hline Brisbane & $2(5 \%)$ \\
\hline Non-metropolitan & $4(9 \%)$ \\
\hline \multicolumn{2}{|l|}{ Western Australia } \\
\hline Perth & $3(7 \%)$ \\
\hline Non-metropolitan & $2(5 \%)$ \\
\hline \multicolumn{2}{|l|}{ South Australia } \\
\hline Adelaide & $2(5 \%)$ \\
\hline Non-metropolitan & $1(2 \%)$ \\
\hline \multicolumn{2}{|l|}{ Northern Territory } \\
\hline Darwin & $0(0 \%)$ \\
\hline Non-metropolitan & $1(2 \%)$ \\
\hline \multicolumn{2}{|c|}{ Australian Capital Territory } \\
\hline Canberra & $1(2 \%)$ \\
\hline Non-metropolitan & $0(0 \%)$ \\
\hline Tasmania & $0(0 \%)$ \\
\hline \multicolumn{2}{|l|}{ New Zealand } \\
\hline Auckland & $1(2 \%)$ \\
\hline Non-metropolitan & $1(2 \%)$ \\
\hline \multicolumn{2}{|c|}{ Australia and New Zealand } \\
\hline State capital city & $28(66 \%)$ \\
\hline Non-metropolitan & $14(34 \%)$ \\
\hline Total & 42 \\
\hline
\end{tabular}

\section{Diagnosis of cicatrising conjunctivitis}

Seventy-seven percent $(n=27 / 35)$ of patients underwent a conjunctival biopsy in the initial diagnostic investigation. Seventy-eight percent $(n=14 / 18)$ of the patients with OcMMP had a conjunctival biopsy to confirm diagnosis. Thirty-three cases had documented data on the duration of symptoms prior to diagnosis. Diagnostic delay ranged from less than a day to over 20 years for cicatrising conjunctivitis, with a mean duration of ocular symptoms when first seen by an ophthalmologist of 2.7 years (median, 3 months). Of the four cases of SJS/TEN, diagnostic delay was significantly shorter, with a mean duration of 12.8 days (range, 2-28 days). Thirty percent of patients $(n=10 / 33)$ had symptoms persisting for over a year prior to diagnosis and a further $25 \%(n=8 / 33)$ had a diagnostic delay greater than or equal to three months. Of these patients, $11(61 \%)$ had OcMMP and the remaining seven underlying diagnoses were unrecorded. The mean duration of diagnostic delay for patients with OcMMP that had extraocular manifestations of their disease was 184 days compared with 1763 days for those without preceding extraocular manifestations.

\section{Clinical features}

At initial presentation, $26 \%$ of patients $(n=9 / 35)$ had moderate or severe conjunctival inflammation, $51 \%(n=$ $18 / 35)$ had symblepharon formation and $14 \%(n=5 / 35)$ had ankyloblepharon on clinical examination (Table 3). At the twelve-month follow-up, $22 \%$ of patients $(n=6 / 27)$ had moderate or severe conjunctival inflammation, $63 \%(n=$ 17/27) had symblepharon formation and 26\% $(n=7 / 27)$ had anklyoblepharon.

At diagnosis, $14 \%$ of patients $(n=5 / 35)$ had bestcorrected visual acuities (BCVAs) of less than 6/18, with no comorbid ocular disease to explain the impaired vision in three of these patients. A sixth patient experienced deterioration in their vision to less than $6 / 18$ at the 12-month follow-up. Four of the 27 patients with recorded data at the 12-month follow-up period that were initially documented as having OcMMP based on clinical phenotype were recorded as 'unknown' underlying aetiology at last visit due to conjunctival biopsies yielding negative results.

\section{Choice of therapy}

The choice of systemic and topical treatment varied between reporting clinicians. There was no statistically significant correlation between the choice of treatment at initial presentation and progression of BCVA or slit-lamp examination findings at the 12-month follow-up. Twentytwo patients $(n=22 / 35,63 \%)$ were managed with systemic immunosuppression or immunomodulation at initial diagnosis. The most commonly utilised therapies included corticosteroids $(n=7 / 22,32 \%)$, methotrexate $(n=4 / 22,18 \%)$, doxycycline $(n=4 / 22,18 \%)$, dapsone $(n=3 / 22,14 \%)$ and sulphapyridine $(n=3 / 22,14 \%)$. Two patients $(9 \%)$ were each treated with intravenous immunoglobulin. Sulfasalazine, cyclophosphamide, azathioprine and mycophenolate were utilised in a single case each (5\%). Seven patients (32\%) received a combination of two or more systemic medications. At the 12-month follow-up, 37\% $(n=12 / 27)$ of patients underwent changes to their systematic therapy, which included two patients in whom treatment was ceased due to adverse effects or lack of compliance.

Topical therapies were recorded for 34 patients ( $n=34 / 35,97 \%)$ at diagnosis. Of the topical medications initiated, 29\% $(n=10 / 34)$ were preservative-free, $47 \%$ ( $n=16 / 34)$ contained preservatives, and the remaining $24 \%$ 
Table 3 Clinical features of patients with cicatrising conjunctivitis at initial diagnosis

\begin{tabular}{|c|c|c|c|c|}
\hline & OcMMP (\%) & SJS/TEN (\%) & $\begin{array}{l}\text { Other CC causes } \\
(\%)\end{array}$ & Total $(\%)$ \\
\hline $\mathrm{BCVA}<6 / 18$ in one or both eyes & $2 / 18(11 \%)$ & $3 / 4(75 \%)$ & $0 / 13(0 \%)$ & $5 / 35(14 \%)$ \\
\hline $\begin{array}{l}\text { Conjunctival inflammation (moderate/ } \\
\text { severe) }\end{array}$ & $4 / 18(22 \%)$ & $1 / 4(25 \%)$ & $4 / 13(31 \%)$ & $9 / 35(26 \%)$ \\
\hline \multicolumn{5}{|l|}{ Lids/lashes: } \\
\hline Trichiasis & $7 / 18(39 \%)$ & $0 / 4(0 \%)$ & $4 / 13(31 \%)$ & $\begin{array}{l}11 / 35 \\
(31 \%)\end{array}$ \\
\hline Cicatrical entropion & $4 / 18(22 \%)$ & $0 / 4(0 \%)$ & $3 / 13(23 \%)$ & $7 / 35(20 \%)$ \\
\hline Symblepharon formation & $8 / 18(44 \%)$ & $1 / 4(25 \%)$ & $9 / 13(69 \%)$ & $\begin{array}{l}18 / 35 \\
(51 \%)\end{array}$ \\
\hline Anklyoblepharon & $5 / 18(28 \%)$ & $0 / 4(0 \%)$ & $0 / 13(0 \%)$ & $5 / 35(14 \%)$ \\
\hline \multicolumn{5}{|l|}{ Lower fornix shrinkage } \\
\hline $25 \%$ or less & $6 / 18(33 \%)$ & $1 / 4(25 \%)$ & $1 / 13(8 \%)$ & $8 / 35(23 \%)$ \\
\hline $25-50 \%$ & $5 / 18(28 \%)$ & $0 / 4(0 \%)$ & $7 / 13(54 \%)$ & $\begin{array}{l}12 / 35 \\
(34 \%)\end{array}$ \\
\hline $50-75 \%$ & $3 / 18(17 \%)$ & $1 / 4(25 \%)$ & $2 / 13(15 \%)$ & $5 / 35(14 \%)$ \\
\hline Obliteration of the fornix & $1 / 18(6 \%)$ & $0 / 4(0 \%)$ & $0 / 13(0 \%)$ & $1 / 35(3 \%)$ \\
\hline \multicolumn{5}{|l|}{ Keratinisation of the ocular surface } \\
\hline Partial & $1 / 18(6 \%)$ & $1 / 4(25 \%)$ & $2 / 13(15 \%)$ & $4 / 35(11 \%)$ \\
\hline Full & $1 / 18(6 \%)$ & $0 / 4(0 \%)$ & $0 / 13(0 \%)$ & $1 / 35(3 \%)$ \\
\hline \multicolumn{5}{|l|}{ Keratopathy } \\
\hline Persistent epithelial defect/s & $1 / 18(6 \%)$ & $1 / 4(25 \%)$ & $0 / 13(0 \%)$ & $2 / 35(6 \%)$ \\
\hline Limbitis \pm peripheral scarring & $3 / 18(17 \%)$ & $1 / 4(25 \%)$ & $1 / 13(8 \%)$ & $5 / 35(14 \%)$ \\
\hline Hx microbial keratitis & $1 / 18(6 \%)$ & $0 / 4(0 \%)$ & $0 / 13(0 \%)$ & $1 / 35(3 \%)$ \\
\hline Central opacities \pm vascularisation & $2 / 18(11 \%)$ & $1 / 4(25 \%)$ & $2 / 13(15 \%)$ & $5 / 35$ \\
\hline
\end{tabular}

$B C V A$ best-corrected visual acuity, $C C$ cicatrising conjunctivitis, $S J S$ Steven-Johnson syndrome, $T E N$ toxic epidermal necrolysis $(n=9 / 34)$ were treated with a combination of both compositions. The majority of patients $(n=27 / 34,79 \%)$ received lubricant drops, gel or ointment, whilst seven patients $(21 \%)$ did not receive any topical lubricant therapy. Twenty patients $(59 \%)$ were treated with topical steroids and three patients (9\%) received topical glaucoma medications. Less commonly utilised topical therapies included autologous serum, minomycin, chloramphenicol, $0.05 \%$ cyclosporine and prednisolone eyedrops.

\section{Discussion}

This surveillance study is the first to prospectively document the nature and incidence of cicatrising conjunctivitis in Australia and New Zealand and the second such study worldwide. The mean age of patients was 74 years, with a significantly lower average age of 43 years for those diagnosed with SJS/TEN, consistent with its pathogenesis as a cutaneous drug hypersensitivity reaction [15]. There was a slight predominance of males reported overall $(n=57 \%)$. This was consistent with Radford and colleagues' (2012) study of cicatrising conjunctivitis in the United Kingdom, in which a greater proportion of patients were male (except in the SJS/TEN sub-group in which more patients were female), suggesting that gender could be a risk factor for certain aetiologies of cicatrising conjunctivitis.

Based on population data, the minimum incidence of cicatrising conjunctivitis was calculated at $1.5 /$ million in Australia and New Zealand (0.7/million for OcMMP), comparable to the incidence of $1.3 /$ million recorded in the United Kingdom (0.8/million for OcMMP) [12]. The most common aetiology of cicatrising conjunctivitis in our study was OcMMP (51.4\%), followed by SJS/TEN (11.4\%), GVHD $(8.6 \%)$ and medication-induced cicatrising conjunctivitis (5.7\%). In the United Kingdom, Radford and colleagues (2012) also recorded OcMMP as the most common cause of cicatrising conjunctivitis ( $61 \%$ of cases), with $20 \%$ attributable to SJS/TEN and $20 \%$ to alternate aetiologies [12]. Incidence data were in keeping with the 1.13 /million incidence of systemic MMP recorded in a prospective study of patients presenting to dermatologists with subepidermal bullous diseases in three regions of France [16], noting that $70 \%$ of these patients would be expected to develop ocular manifestations [5]. A lower incidence of $0.87 /$ million for cicatricial pemphigoid was 
published in Germany, based only on patients presenting to one dermatology referral centre in central Germany [17]. This suggests that predisposition for OcMMP does not significantly vary between these developed countries. The studies from France and Germany, however, were obtained from prospective studies targeting particular geographical regions and patient subgroups, which potentially contributed to selection biases.

More recently, Hubner and colleagues (2016) analysed prevalence data based on the records of a major German health insurance company, which represented approximately $12 \%$ of the total German population from different geographical regions [18], thereby likely involving less selection bias than the prior French and German incidence studies. The prevalence of pemphigus and pemphigoid diseases was calculated at $0.05 \%$ and the prevalence of MMP at 24.6/million, with a higher proportion of females than males affected [18]. If $70 \%$ of these patients are expected to develop ocular manifestations [5], the prevalence of OcMMP could be estimated at 17.2/million. Notably, is difficult to make direct comparisons given that this study measures prevalence rather than incidence and is also based on German data. However, it still provides an important context in linking prevalence and incidence data given that OcMMP is considered a chronic disease where maintenance treatment typically permits sustained survival [3-5]. It could thus be inferred that incidence contributes to growth of prevalence until the mortality equals or exceeds the incidence rate.

The incidence of cicatrising conjunctivitis and OcMMP varied in their geographical distribution within Australia and New Zealand. Melbourne (Victoria) had the highest incidence of any state capital city at $1.8 /$ million compared with Sydney (New South Wales) at $0.5 /$ million, despite their relatively similar ethnic demographic and population size compared with other Australian state capital cities. While $26 \%$ of the reporting centres were located in Sydney compared to $19 \%$ in Melbourne (Tables 1, 2), Melbourne has specialist clinics for OcMMP that were actively reporting new diagnoses to the ANZOSU, which may have contributed to the higher incidence rates. Although the Northern Territory had the highest incidence of any state or territory at 3.3/million, this was represented by only one reported case in a town within this state, making it difficult to draw reliable comparisons about the higher incidence rate.

The small population of a number of non-metropolitan regions in which positive cases were reported and the significant variations in population size between postal areas also led to disproportionately high recorded incidences in these areas, including Alice Springs, Augusta, Horsham and Lismore/Byron Bay (Table 1). Using postal codes as a geographical identifier in the questionnaires also had other limitations. Postal codes do not always correlate with postal areas, which vary in size, change over the years and may overlap [19]. Furthermore, the postal codes listed by reporting ophthalmologists may have been patients' postal rather than residential addresses, leading to potential inaccuracies. In future surveillance studies, requesting the patient's residential city or town and state would provide more accurate data, however, may be limited by privacy issues and ethics approval.

Varying levels of reporting amongst ophthalmologists, lack of awareness of cicatrising conjunctivitis, and potentially different genetic susceptibilities may have contributed to the geographical variation in incidence. The 56 cases of cicatrising conjunctivitis were reported by 47 different ophthalmologists at 42 different centres across Australia and New Zealand, with the majority of reporting centres $(66 \%)$ located in state capital cities. However, an equal proportion of the patients with cicatrising conjunctivitis resided in metropolitan and nonmetropolitan regions. This suggests that patients may have travelled to state capital cities for diagnosis. While the data lends support to establishing more regional specialist centres for cicatrising conjunctivitis, it is difficult to draw conclusions from direct comparison of the location of reporting centres and residence of reported patients due to missing geographical patient data for 21 of the 56 reported cases.

Ocular MMP is typically diagnosed based upon a characteristic phenotype, direct immunofluorescence (DIF) or immunohistochemistry on conjunctival biopsy and/or the presence of autoantibodies on indirect immunofluorescence [1, 12, 20-22]. Four of the cases initially diagnosed as OcMMP had a negative DIF biopsy result at the 12-month follow-up. Notably, DIF is neither specific nor sensitive for OcMMP and false negative biopsy results have previously been reported, contributing to delayed recognition and disease progression. A case series of 49 patients with OcMMP showed that whilst $51 \%$ of patients had positive immunofluorescence test results, $26 \%$ required more than 1 biopsy to yield a positive result [1, 10, 20, 23]. Dart and colleagues (2017) proposed a revised criteria in which the diagnosis of OcMMP can be made based upon a typical phenotype of progressive conjunctival scarring and exclusion of other causative conditions despite negative immunopathology results [1]. According to these criteria, while four reporting ophthalmologists in our study recorded a different diagnosis at the 12-month follow-up due to negative biopsy results, the patients could still have had presumed OcMMP. Until a more sensitive marker is identified, increased awareness of the limitations of immunological testing in OcMMP amongst ophthalmologists is necessary to reduce misdiagnosis and delayed treatment. 
The mean diagnostic delay for cicatrising conjunctivitis in our surveillance study was 2.7 years (range, 0 days-20 years). Patients with an underlying aetiology of SJS/TEN had a significantly shorter duration of ocular symptoms prior to diagnosis of 12.8 days (range, 2-28 days). This is in line with the more acute onset and progression of SJS/TEN compared with OcMMP and other aetiologies of cicatrising conjunctivitis [5, 15]. Notably, the diagnostic delay for patients with OcMPP that had no preceding extraocular manifestations was significantly greater than those with preceding extraocular manifestations. This was comparable with data on diagnostic delay in the United Kingdom's surveillance of cicatrising conjunctivitis [13], and suggests that patients with ocular-only MMP may experience initial misdiagnosis of their symptoms and delays in treatment, potentially secondary to difficulty recognising the condition. Therefore, it is important to consider OcMMP in cases of recurrent conjunctivitis and trichiasis, remembering that a negative conjunctival biopsy cannot exclude OcMMP [1, $10,20,23]$. Increased awareness of the condition amongst general ophthalmologists and referral to specialist centres should be prioritised.

Disease progression over the 12-month follow-up period demonstrated similar levels of conjunctival inflammation $(26 \%$ with moderate or severe inflammation at diagnosis to $22 \%$ at 12 months) and an increase in symblepharon and anklyblepharon formation. Sixty-three percent of patients were commenced on systemic immunosuppressive therapy at diagnosis, which correlates closely with the $64 \%$ in Radford and colleagues' (2012) British study [12]. This suggests similarities in the approach to initial treatment between the countries, and may also indicate a reluctance to use immunosuppression outside large specialist centres [1, 12].

Similar to the surveillance study recently conducted by our group via the ANZOSU on LSCD, the current study is limited by incomplete inclusion of practicing ophthalmologists in the ANZOSU database and their response rates. We recently reported a $38 \%$ inclusion rate of practising ophthalmologists in the ANZOSU database and a $62 \%$ response rate of ophthalmologists returning ANZOSU report cards [13]. Given that the incidence study on cicatrising conjunctivitis was conducted 1-2 years earlier, it is likely that the inclusion and response rates were similar, or even lower than the LSCD study. The difficulty in subsequently obtaining completed questionnaires from reporting ophthalmologists (incomplete or erroneous data for $36 \%$ of positive cases and $77 \%$ completion rate of follow-up questionnaires) may also have skewed the results, particularly the geographical data. Unfortunately, poor response rates are an inherent limitation of most non-compulsory surveillance studies. Notably, as a RANZCO-affiliated Unit, the ANZOSU is likely to have achieved better response rates than the alternative approach of investigators contacting ophthalmologists directly to report positive cases. Utilising electronic questionnaires rather than paper forms, recruiting more corneal specialists to the study, and increasing the duration of surveillance could improve response rates in the future [14]. Unfortunately, due to the disbanding of the ANZOSU, inclusion and response rates for surveillance studies of ocular diseases in Australia and New Zealand may continue to be a limiting factor. While patient-reported data through the Australian Bureau of Statistics or a similarly reputable organisation is a possibility, the complexity of cicatrising conjunctivitis and difficulty in diagnosis even amongst ophthalmologists makes this a less pragmatic option.

Despite its limitations, the surveillance study provides an estimate of the minimum incidence of cicatrising conjunctivitis that is in keeping with Radford and colleagues' (2012) national incidence data in the United Kingdom and other smaller-scale French and German studies [12, 16, 17]. As the second nationwide prospective study on cicatrising conjunctivitis conducted worldwide, it provides useful data for comparative analysis internationally, as well as novel information on the geographical distribution, diagnostic delay, and management approaches of cicatrising conjunctivitis within Australia and New Zealand.

\section{Summary}

\section{What was known before:}

- Cicatrising conjunctivitis is a rare potentially sightthreatening condition characterised by persistent conjunctival inflammation and progressive conjunctival scarring.

- In the United Kingdom, the minimum incidence of cicatrising conjunctivitis was calculated as of 1.3/ million, including 0.8/million for ocular MMP.

\section{What this study adds:}

- This is the second nation-wide prospective surveillance study worldwide to document the incidence of cicatrising conjunctivitis and the first in Australia and New Zealand.

- It provides useful data for international comparison, as well as novel information on the geographical distribution, diagnostic delay and management approaches of cicatrising conjunctivitis.

Acknowledgements NHMRC Career Development Fellowship for Author SW (APP1050524). Professor Watson is also supported by the Sydney Medical School Foundation. Australian and New Zealand Ophthalmic Surveillance Unit. Valerie P. Saw FRCOphth, Moorfields 
Eye Hospital, London, United Kingdom-collaboration in study design. Geraint P. Williams FRCOphth, Academic Unit of Ophthalmology, University of Birmingham, Birmingham, United Kingdomcollaboration in study design. Saaehla Rauz FRCOphth, Academic Unit of Ophthalmology, University of Birmingham, Birmingham, United Kingdom - collaboration in study design. Cherry Radford FRCOphth, Moorfields Eye Hospital, NHS Foundation Trust, City Road, London, United Kingdom-collaboration in study design. John Dart FRCOphth, Moorfields Eye Hospital, NHS Foundation Trust, City Road, London, United Kingdom-collaboration in study design.

Funding NHMRC Career Development Fellowship for Author SW (APP1050524)

\section{Compliance with ethical standards}

Conflict of interest The authors declare that they have no conflict of interest.

\section{References}

1. Dart JK. The 2016 Bowman Lecture Conjunctival curses: scarring conjunctivitis 30 years on. Eye. 2017;31:301-32.

2. Williams GP, Radford C, Nightingale P, Dart JK, Rauz S. Evaluation of early and late presentation of patients with ocular mucous membrane pemphigoid to two major tertiary referral hospitals in the United Kingdom. Eye. 2011;25:1207-18.

3. Elder MJ, Bernauer W, Leonard J, Dart JK. Progression of disease in ocular cicatricial pemphigoid. Br J Ophthalmol. 1996;80: 292-6.

4. Chan LS, Ahmed AR, Anhalt GJ, Bernauer W, Cooper KD, Elder MJ, et al. The first international consensus of mucous membrane pemphigoid-definition, diagnostic criteria, pathogenic factors, medical treatment, and prognostic indicators. Arch Dermatol. 2002;138:370-9.

5. Saw VP, Dart JK. Ocular mucous membrane pemphigoid: diagnosis and management strategies. Ocul Surf. 2008;6:128-42.

6. Tauber J, Sainz DLM, Foster CS. Systemic chemotherapy for ocular cicatricial pemphigoid. Cornea. 1991;10:185-95.

7. Saw VP, Dart JK, Rauz S, Ramsay A, Bunce C, Xing W, et al. Immunosuppressive therapy for ocular mucous membrane pemphigoid: strategies and outcomes. Ophthalmology. 2008;115: 253-61.

8. Thorne JE, Woreta FA, Jabs DA, Anhalt GJ. Treatment of ocular mucous membrane pemphigoid with immunosuppressive drug therapy. Ophthalmology. 2008;115:2146-52.

9. Bernauer W, Broadway DC, Wright P. Chronic progressive conjunctival cicatrisation. Eye. 1993;7:371-8.
10. Thorne JE, Anhalt GJ, Jabs DA. Mucous membrane pemphigoid and pseudopemphigoid. Ophthalmology. 2004;111:45-52.

11. Shikari H, Antin JH, Dana R. Ocular graft-versus-host disease: a review. Surv Ophthalmol. 2013;58:233-51.

12. Radford CF, Rauz S, Williams GP, Saw VP, Dart JK. Incidence, presenting features, and diagnosis of cicatrising conjunctivitis in the United Kingdom. Eye. 2012;26:1199-208.

13. Bobba S, Di Girolamo N, Mills R, Daniell M, Chan E, Harkin DG, et al. Nature and incidence of severe limbal stem cell deficiency in Australia and New Zealand. Clin Exp Ophthalmol. 2017;45:174-81.

14. Foot B, Stanford M, Rahi J, Thompson J. The British Ophthalmological Surveillance Unit: an evaluation of the first 3 years. Eye. 2003;17:9-15.

15. Hazin R, Ibrahimi OA, Hazin MI, Kimyai-Asadi A. StevensJohnson syndrome: Pathogenesis, diagnosis, and management. Ann Med. 2008;40:129-38.

16. Bernard P, Vaillant L, Labeille B, Bedane C, Arbeille B, Denoeux JP, et al. Incidence and distribution of subepidermal autoimmune bullous skin diseases in three French regions. Bullous Diseases French Study Group. Arch Dermatol. 1995;131: $48-52$.

17. Zillikens D, Wever S, Roth A, Weidenthaler-Barth B, Hashimoto $\mathrm{T}$, Brocker EB. Incidence of autoimmune subepidermal blistering dermatoses in a region of central Germany. Arch Dermatol. $1995 ; 131: 957-8$

18. Hubner F, Recke A, Zillikens D, Linder R, Schmidt E. Prevalence and age distribution of pemphigus and pemphigoid diseases in Germany. J Invest Dermatol. 2016;136:2495.

19. Australian Bureau of Statistics. Australian Statistical Geography Standard (ASGS): Volume 3 - Non ABS Structures, July 2011. Canberra: Australian Bureau of Statistics; 2011 Jul. Accessed 10/ 10/2017, available at http://www.abs.gov.au/ausstats/abs@.nsf/0/ 736A18F4CBF81595CA2578D40012CF51.

20. Bernauer W, Elder MJ, Leonard JN, Wright P, Dart JK. The value of biopsies in the evaluation of chronic progressive conjunctival cicatrisation. Graefes Arch Clin Exp Ophthalmol. 1994;232: 533-7.

21. Power WJ, Neves RA, Rodriguez A, Dutt JE, Foster CS. Increasing the diagnostic yield of conjunctival biopsy in patients with suspected ocular cicatricial pemphigoid. Ophthalmology. 1995;102:1158-63.

22. Grau AE, Setterfield J, Saw VP. How to do conjunctival and buccal biopsies to investigate cicatrising conjunctivitis: improving the diagnosis of ocular mucous membrane pemphigoid. $\mathrm{Br} \mathrm{J}$ Ophthalmol. 2013;97:530-1.

23. Leonard JN, Hobday CM, Haffenden GP, Griffiths CE, Powles $\mathrm{AV}$, Wright $\mathrm{P}$, et al. Immunofluorescent studies in ocular cicatricial pemphigoid. Br J Dermatol. 1988;118:209-17. 